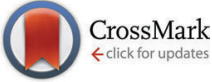

Cite this: Phys. Chem. Chem. Phys., 2015, 17, 13937

Received 19th February 2015, Accepted 29th April 2015

DOI: $10.1039 / c 5 c p 01045 d$

www.rsc.org/pccp

\section{High-resolution X-ray absorption spectroscopy of iron carbonyl complexes $\dagger$}

\author{
Andrew J. Atkins, ${ }^{a}$ Matthias Bauer ${ }^{\star b}$ and Christoph R. Jacob*ac \\ We apply high-energy-resolution fluorescence-detected (HERFD) X-ray absorption near-edge spectroscopy \\ (XANES) to study iron carbonyl complexes. Mono-, bi-, and tri-nuclear carbonyl complexes and pure \\ carbonyl complexes as well as carbonyl complexes containing hydrocarbon ligands are considered. The \\ HERFD-XANES spectra reveal multiple pre-edge peaks with individual signatures for each complex, which \\ could not be detected previously with conventional XANES spectroscopy. These peaks are assigned and \\ analysed with the help of TD-DFT calculations. We demonstrate that the pre-edge peaks can be used to \\ distinguish the different types of iron-iron interactions in carbonyl complexes. This opens up new possibilities \\ for applying HERFD-XANES spectroscopy to probe the electronic structure of iron catalysts.
}

\section{Introduction}

Iron carbonyl complexes are the text-book example of coordination compounds following the 18 electron rule. The metal centre(s) coordinates to a set of ligands which will provide enough electrons to fill the valence shell. ${ }^{1-3}$ Here, the necessary 18 electrons can also be reached by forming a (formal) metalmetal bond in multinuclear carbonyls. Additionally, carbonyls are the prototypical example for synergetic effects by $\sigma$-donation and $\pi$-backdonation from the CO ligand to the metal centre(s) and vice versa. The ability to spectroscopically probe the changes in the electronic structure caused by the binding of CO ligands as well as by the formation of metal-metal bonds is key for understanding catalytic reactions featuring iron carbonyls or other types of iron clusters as active species.

Examples of such reactions are the Fischer-Tropsch process ${ }^{4-10}$ or the CO oxidation reaction. ${ }^{11,12}$ Moreover, iron carbonyl complexes are of interest as model complexes to understand the working principles of catalysts. ${ }^{13-15}$ Finally, iron carbonyls have also been identified as potential substitutes to replace expensive catalysts based on rare elements such as ruthenium or platinum. ${ }^{16}$

The catalytic activity of iron carbonyls is usually attributed to two factors: First, the ease of the (photo-)dissociation of a carbonyl ligand in the iron carbonyl complexes, ${ }^{15,17,18}$ and second,

\footnotetext{
${ }^{a}$ Karlsruhe Institute of Technology (KIT), Center for Functional Nanostructures and Institute of Physical Chemistry, Wolfgang-Gaede-Str. 1a, 76131 Karlsruhe, Germany

${ }^{b}$ Universität Paderborn, Naturwissenschaftliche Fakultät, Department Chemie, Warburger Straße 100, 33098 Paderborn, Germany. E-mail: matthias.bauer@upb.de ${ }^{c}$ TU Braunschweig, Institute of Physical and Theoretical Chemistry,

Hans-Sommer-Str. 10, 38106 Braunschweig, Germany.

E-mail: c.jacob@tu-braunschweig.de

$\dagger$ Electronic supplementary information (ESI) available: Additional calculated X-ray absorption spectra. See DOI: 10.1039/c5cp01045d
}

the weak bonding character of the Fe-Fe contact that is easily broken. ${ }^{19}$ Both pathways lead to coordinatively unsaturated species which undergo fast interactions with reactants. However, it is often difficult - if not impossible - to study the bonding of $\mathrm{CO}$ and the iron-iron bond in the course of chemical reactions. Although several spectroscopic studies on iron carbonyls exist (see, e.g., ref. 18, 20 and 21), these spectroscopic methods can hardly be used under real reaction conditions (high pressure and temperatures) due to vacuum requirements for X-ray and ultraviolet photoelectron spectroscopy (XPS and UPS), ${ }^{22,23}$ due to low penetration depth or the interference of solvents for infrared (IR) spectroscopy, or simply the fact that window materials might not be transparent for the radiation used.

Hard X-ray absorption spectroscopy offers a unique opportunity to overcome these limitations by using high-energy radiation in an element specific manner. ${ }^{24}$ Previously, it was shown that valence-to-core X-ray emission spectroscopy (V2C-XES) can be used to identify details of the ligand coordination as well as the substitution of CO by hydrocarbons. ${ }^{25}$ However, since V2C-XES spectra are dominated by the ligand levels, details of the metalmetal interaction can barely be accessed.

X-ray absorption near edge structure (XANES) spectroscopy can provide information on the qualitative or comparative oxidation states in a fingerprinting way by comparing the absorption edge position of the sample with well-defined references. However, with respect to probing the electronic structure of iron carbonyls during catalytic reactions, much of the information on the geometric and electronic structure is contained in the pre-edge signal. In the case of iron K-edge spectra, pre-edge peaks are caused by 1s $\rightarrow 3 \mathrm{~d}$ transitions, ${ }^{26-28}$ which thus probe the lowest unoccupied molecular orbitals (LUMOs) of metal catalysts. These unoccupied states reflect the geometric (coordination symmetry) and electronic (oxidation state) structure. However, extracting this information 
<smiles></smiles>

(a)

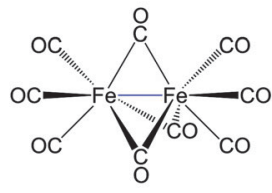

(b)

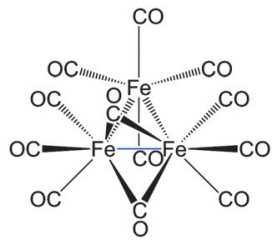

(c)

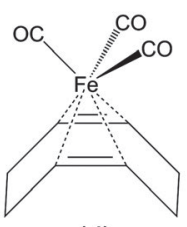

(d)

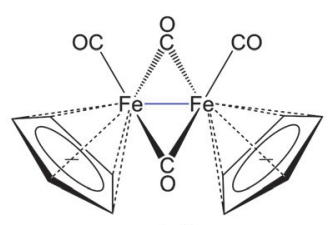

(e)
Fig. 1 Lewis structures of the five iron carbonyl complexes considered in this work. (a) $\mathrm{Fe}(\mathrm{CO})_{5}$, (b) $\mathrm{Fe}_{2}(\mathrm{CO})_{9}$, (c) $\mathrm{Fe}_{3}(\mathrm{CO})_{12}$, (d) $\mathrm{Fe}(\mathrm{CO})_{3}\left(\mathrm{C}_{8} \mathrm{H}_{12}\right)$, and (e) $\mathrm{Fe}_{2}(\mathrm{CO})_{4}(\mathrm{Cp})_{2}$.

from conventional transition metal K-edge XANES spectra is complicated by the core-hole lifetime broadening of the $1 \mathrm{~s}$ electron hole, which smears out features in the pre-edge region.

Eisenberger et $a .^{29}$ showed that the lifetime broadening in the XANES region could be reduced by detecting the X-ray absorption spectrum using the intensity of the emitted X-ray fluorescence in a narrow energy bandwidth. This technique was called high-energy-resolution fluorescence detected XANES (HERFDXANES). ${ }^{30-32}$ Previously we have shown that HERFD-XANES can reveal subtle changes in the electronic structure of iron complexes that are not detectable with V2C-XES spectroscopy. ${ }^{33,34}$ In this work, we want to establish the benefits of HERFD-XANES spectroscopy for the investigation of multinuclear metal complexes in general and iron carbonyls in particular.

To this end, we investigate the HERFD-XANES spectra of iron carbonyl with a special focus on the identification of signatures characteristic of CO-bonding and different types of $\mathrm{Fe}-\mathrm{Fe}-\mathrm{bond}$ as well as their change due to ligand exchange. We use a test set of five iron carbonyl complexes consisting of (a) iron-pentacarbonyl $\mathrm{Fe}(\mathrm{CO})_{5}$, (b) di-iron-nonacarbonyl $\mathrm{Fe}_{2}(\mathrm{CO})_{9}$, (c) tri-iron-dodecacarbonyl $\mathrm{Fe}_{3}(\mathrm{CO})_{12}$, (d) iron-tricarbonyl-cyclooctadiene $\mathrm{Fe}(\mathrm{CO})_{3}\left(\mathrm{C}_{8} \mathrm{H}_{12}\right)$, and (e) di-iron-tetracarbonyl-dicyclopentadiene $\mathrm{Fe}_{2}(\mathrm{CO})_{4}(\mathrm{Cp})_{2}$. The corresponding Lewis structures are shown in Fig. 1.

This work is organised as follows. In Section 2, we introduce the experimental and computational methodology used for recording and calculating the pre-edge region of the HERFDXANES spectra. This is followed by a discussion of the electronic structure and spectra of $\mathrm{Fe}(\mathrm{CO})_{5}$ in Section 3. In Sections 4 and 5, the multinuclear iron carbonyls as well as substituted iron carbonyls are discussed, respectively. Finally, the results are summarised and conclusions are drawn in Section 6.

\section{Methodology}

\subsection{Experimental}

The samples were purchased from Sigma Aldrich in high purity and used without further purification. The solid samples (b)-(e) were diluted with $\mathrm{BN}$ in a ratio of approximately $1: 4$. For the liquid $\mathrm{Fe}(\mathrm{CO})_{5}$, $\mathrm{BN}$ was impregnated with a corresponding amount. All sample preparation was carried out under an inert atmosphere in a glove box.

All spectra were recorded using a scanning Johann ${ }^{35,36}$ type spectrometer as available at the ESRF Grenoble, beamline ID26. ${ }^{37}$ Data acquisition was carried out in a horizontal plane, where the sample, crystal analysers and photon detector (avalanche photodiode) were arranged in a vertical Rowland geometry. An incident flux of $\approx 2 \times 10^{13}$ photons per $\mathrm{s}$ is estimated at this experimental station. The incident energy was selected using the (311) reflection from a Si double crystal monochromator. To record HERFD-XANES spectra, the intensity of a single fluorescence channel, selected with the analyser crystals, is monitored with a resolution smaller than the lifetime broadening. Meanwhile, the incident energy of the double crystal monochromator is swept over the edge. Here, the emission energy of $7.056 \mathrm{keV}$ was selected using the (620) reflection of five spherically bent Ge crystal analysers (with $R=1 \mathrm{~m}$ ). The total fluorescence yield (TFY) was monitored by a photodiode. Measurements were carried out at $15 \mathrm{~K}$ using a He cryostat under vacuum conditions. To exclude radiation damage, fast measurements over the energy region 7110-7146 eV with a scan-time of four seconds were carried out before and after the HERFD-XANES acquisition. No radiation damage was detected in this procedure. Energy calibration of the monochromator was performed using Fe foil. The emission spectrometer was calibrated using the elastic scattering line. Selected RIXS planes were recorded to exclude off-diagonal signals that could lead to misinterpretations of the HERFD-XANES spectra.

Fig. 2 compares the conventional and HERFD-XANES spectra. The significant increase in resolution and the tremendously sharpened signals in the pre-edge region are obvious. Comparing the conventional spectra it is clear that here the pre-edge peaks look very similar. Only for the more ionic complex $\mathrm{Fe}_{2}(\mathrm{CO})_{4}(\mathrm{Cp})_{2}(\mathrm{e})$,

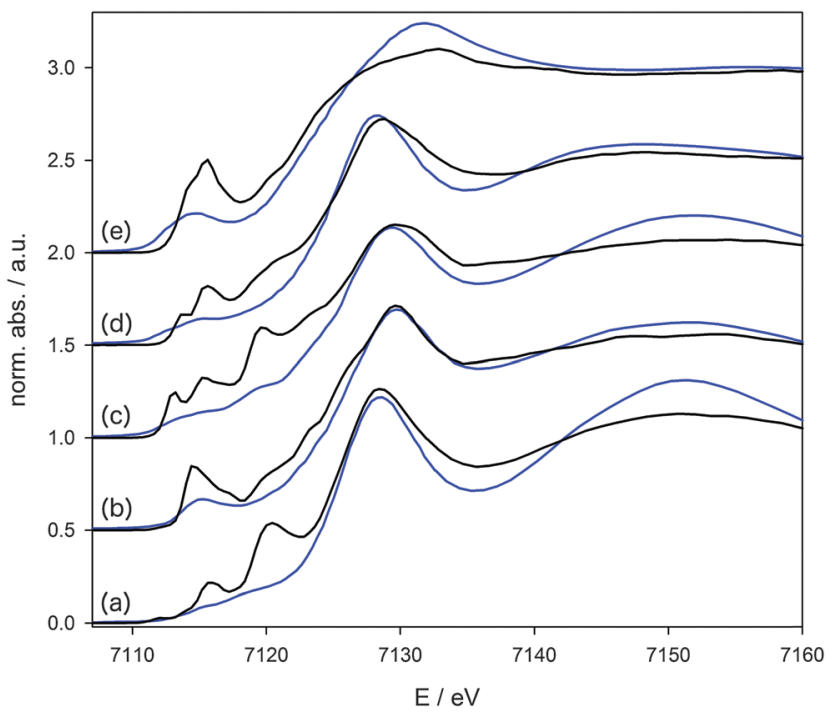

Fig. 2 HERFD-XANES spectra (black solid line) of the compounds (a) Fe(CO) (b) $\mathrm{Fe}_{2}(\mathrm{CO})_{9}$, (c) $\mathrm{Fe}_{3}(\mathrm{CO})_{12}$, (d) $\mathrm{Fe}(\mathrm{CO})_{3}\left(\mathrm{C}_{8} \mathrm{H}_{12}\right)$, and (e) $\mathrm{Fe}_{2}(\mathrm{CO})_{4}(\mathrm{Cp})_{2}$ in comparison to the according conventional XANES spectra (blue solid line). 
with iron in the oxidation state $+\mathrm{I}$, a more intense pre-edge peak is observed compared to all other complexes with a formal oxidation state of $\mathrm{Fe}(0)$. Nonetheless differences with respect to the electronic structure are hard to deduce from the experimental spectra alone, because, first, the pre-edge peak cannot be extracted in a reliable manner due to its strong overlap with the main edge, and, second, because the resolution of the experimental data is too low for a direct comparison with calculations.

The differences in the pre-edge peaks of the carbonyl complexes (a)-(e) drastically increase in the HERFD-XANES data compared to the conventional XANES data. The spectra of all five compounds are now characterised by individual signatures that differ to a large extent. Beside the need for a quantitative understanding of the observed manifold of signals, they could also be used on a more simple basis in a fingerprint approach. However, in order to arrive at a clear assignment and to extract information on the unoccupied electronic states, quantumchemical calculations need to be applied.

Deconvolution of the pre-edge region was carried out by a least square fit of the spectra using asymmetric Gaussian peak profiles in order to account for asymmetries in the RIXS plane from which the HERFD-XANES spectra are extracted. In the first iteration, an arctan step function representing the transition of the ejected photoelectron to the continuum was subtracted from the spectra. In the second step, the remaining pre-edge peaks as identified by inspection of the first two derivatives, were fitted. In Fig. 4, 7 and 9, the isolated and deconvoluted experimental pre-edge peaks are shown.

\subsection{Computational}

Geometry optimisations of the molecular complexes were performed with the ADF program package ${ }^{38,39}$ using the BP86 exchange-correlation functional ${ }^{40,41}$ in combination with the TZP Slater-type basis set from ADF's basis set library. ${ }^{42}$ For all considered compounds, the ground state is the closed-shell singlet spin state.

The XANES spectra for the iron carbonyl complexes were then calculated using time-dependent density functional theory (TD-DFT). To selectively target core excitations, the TD-DFT calculations were restricted to excitations originating from the Fe 1s orbitals. ${ }^{43,44}$ Such restricted-channel TD-DFT calculations have been applied extensively for K-edge XANES spectra of transition metal complexes. ${ }^{45-50}$ The effect of the exchange-correlation functional was judged by performing the calculations with both the non-hybrid functional BP86 $6^{40,41}$ and the hybrid functional B3LYP. ${ }^{40,51-53}$ The QZ4P Slater-type basis set ${ }^{42}$ was applied in all TD-DFT calculations. The intensities include second-order contributions due to the magnetic-dipole and electric-quadrupole transition moments, which are calculated using the originindependent methodology described in ref. 54. Spin-orbit coupling, which could give rise to non-zero intensities of singlet-triplet transitions, has not been considered here.

The BP86 spectra were shifted by $180.62 \mathrm{eV}$ and the B3LYP spectra by $150.08 \mathrm{eV}$ for comparison to experiment. These shifts are chosen such that the energy of the first peak in the calculated spectrum of iron-pentacarbonyl agrees with the first peak in the experimental spectrum. While these shifts are rather large, they do not affect the relative position of the peaks. For Fe K-edge X-ray absorption spectroscopy, it has been demonstrated previously that despite the large absolute errors, the selective TD-DFT approach used here can provide relative excitation energies and intensities with an accuracy that allows for a direct comparison with experiment. ${ }^{55}$ The intensity of the calculated spectra of complexes containing more than one core excitation centre were divided by the number of iron atoms to normalise the spectra. For all the calculated spectra, the ionisation threshold is indicated by a blue vertical line in Fig. 4, 7 and 9. Excitations above this threshold, which is determined as the negative of the energy of the 1s core orbital, cannot be calculated reliably in conventional TDDFT calculations. ${ }^{56}$

It has previously been argued, ${ }^{45}$ that for molecules with equivalent core orbitals it is necessary to localise the core hole before performing the TD-DFT calculation. We note that in the present study, all calculations were run without considering the molecular symmetry, which results in localised core orbitals for the bi- and trimetallic complexes.

All orbital isosurface plots in Fig. 3, 5 and 8 were prepared with Molekel ${ }^{57}$ and use an isosurface value of $\pm 0.025 \mathrm{e} \mathrm{bohr}^{-3}$. With the B3LYP hybrid functionals, some excitations are combinations of several orbital transitions. Fig. 5 and 8 shows the corresponding linear combination of unoccupied orbitals. ${ }^{33}$ This is possible because the excitations originate from the same localised Fe 1s core orbitals.

\section{Revisiting the electronic structure of $\mathrm{Fe}(\mathrm{CO})_{5}$}

First, we consider the simplest iron carbonyl complex $\mathrm{Fe}(\mathrm{CO})_{5}$, which has a trigonal bipyramidal structure with $D_{3 \mathrm{~h}}$ symmetry. As a starting point for the discussion of $\mathrm{Fe}(\mathrm{CO})_{5}$, we will consider a schematic representation of its molecular orbital (MO) diagram, which is shown in Fig. 3. For earlier computational investigations of the electronic structure of $\mathrm{Fe}(\mathrm{CO})_{5}$, see, e.g., ref. 58-61. In the $D_{3 \mathrm{~h}}$ point group the d-orbitals of the iron atom belong to three irreducible representations (irreps). The iron $\mathrm{d}_{z^{2}}$-orbital belongs to the totally symmetric $A_{1}{ }^{\prime}$ irrep, whereas the $\mathrm{d}_{x y}$ and $\mathrm{d}_{x^{2}-y^{2}}$ as well as $\mathrm{d}_{x z}$ and $\mathrm{d}_{y z}$ pairs belong to the two degenerate irreps $E^{\prime}$ and $E^{\prime \prime}$, respectively. These combine with the carbonyl ligand orbitals of the same symmetry. The carbonyl $\sigma$-orbitals form two combinations that belong to the irrep $A_{1}{ }^{\prime}$, one combination that belongs to $A_{2}{ }^{\prime \prime}$, and two combinations belonging to $E^{\prime \prime}$. These carbonyl $\sigma$-orbitals are composed of the p-orbitals pointing along the carbonyl bond axes, and thus form the iron-carbonyl $\sigma$-bonds. The carbonyl $\pi^{*}$-orbitals form two combinations that belong to the irreps $A_{2}{ }^{\prime}$ and $A_{2}{ }^{\prime \prime}$, respectively, four combinations belonging to the $E^{\prime}$ irrep, and four combinations belonging to the $E^{\prime \prime}$ irrep. These carbonyl $\pi^{*}$-orbitals are responsible for the iron-CO $\pi$-backbonding.

The five iron d-orbitals combine with the carbonyl $\sigma$ - and $\pi^{*}$-orbital combinations with the matching symmetry. The five highest occupied and the five lowest unoccupied orbitals each 


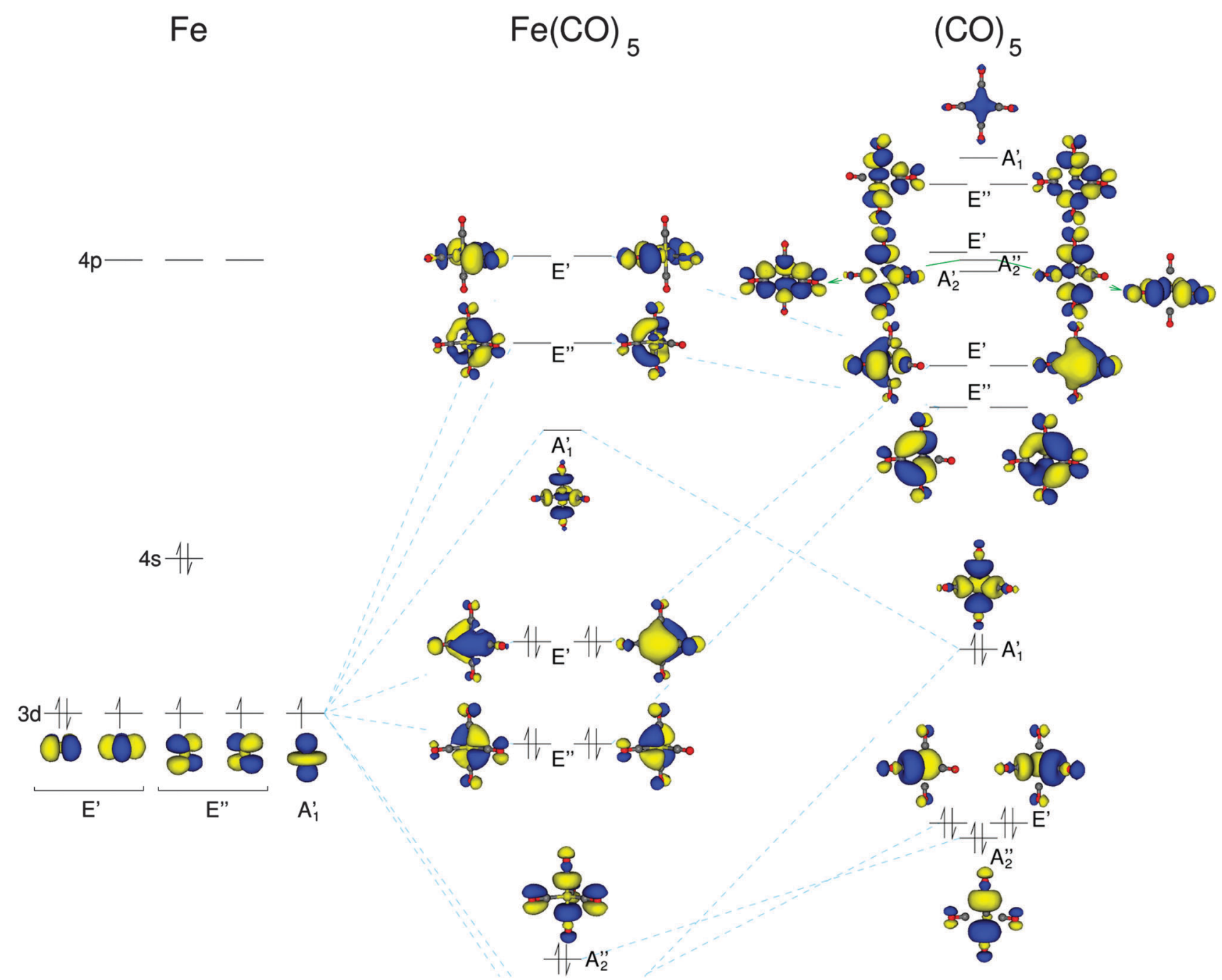

Fig. 3 A schematic molecular orbital diagram of $\mathrm{Fe}(\mathrm{CO})_{5}$ constructed from density-functional theory calculations. The isosurface plots of the occupied and unoccupied orbitals have been obtained with BP86. The orbitals are arranged in the energetical order in which they appear within the B3LYP calculations.

contain the five iron d-orbitals. These molecular orbitals are shown in the MO diagram of Fig. 3. The unoccupied orbitals are those that give rise to the pre-edge peaks in the HERFD-XANES spectra. The iron $\mathrm{d}_{z^{2}}$-orbital interacts with the $A_{1}{ }^{\prime} \sigma$-carbonyl orbitals to form an anti-bonding combination with $A_{1}{ }^{\prime}$ symmetry. The $\mathrm{d}_{x z}$-and $\mathrm{d}_{y z}$-orbitals interact only with the $E^{\prime \prime} \pi$-orbitals of the carbonyls, whereas the $\mathrm{d}_{x y^{-}}$and $\mathrm{d}_{x^{2}-y^{2} \text {-orbitals can interact }}$ with both $\sigma$ and $\pi$ carbonyl orbitals. This can be seen in the unoccupied orbitals of the $E^{\prime}$ symmetry in Fig. 3 where there is some minor inclusion of a $\sigma$ carbonyl orbital on the carbonyl ligand with no $\pi$ contributions, whereas all other carbonyl ligand orbitals are pure $\pi$-orbitals.

Using the character table of point group $D_{3 \mathrm{~h}}$ we can determine whether an X-ray transition from the iron 1s to these unoccupied orbitals will have dipole or quadrupole intensity. Transitions to the $A_{1}{ }^{\prime}$ and $E^{\prime \prime}$ MOs are only quadrupole-allowed, whereas those to $E^{\prime}$ MOs are both quadrupole- and dipole-allowed.

Fig. 4a contains both the calculated and experimental spectra of $\mathrm{Fe}(\mathrm{CO})_{5}$. Therein, the experimental spectrum has three distinct peaks, labeled A, B, and C. Previous experimental X-ray absorption spectra of $\mathrm{Fe}(\mathrm{CO})_{5}$ at the K-edge of iron show only one peak in the pre-edge spectra. ${ }^{62,63}$ This clearly demonstrates the power of HERFD-XANES to reveal the detailed structure of the pre-edge region for transition metal complexes. In the experimental spectrum, peaks A and B clearly belong to the pre-edge. However, peak $\mathrm{C}$, with a significant increase in intensity compared to peaks A and B, is most likely due to dipole-allowed transitions to (Rydberg-like) states close to the absorption edge, which cannot be described with conventional exchange-correlation functionals. ${ }^{64,65}$ Moreover, the region close to and above the ionization threshold will be obscured by quasi-continuum states that cannot be described with finite atom-centered basis sets. ${ }^{56}$ Therefore, we will focus our discussion on peaks A and B in the following.

In the calculated spectra, BP86 shows one intense peak, whereas B3LYP provides a spectrum with three distinct features with lower intensities. Therefore, (qualitatively) we can conclude that the B3LYP exchange-correlation functional provides results 


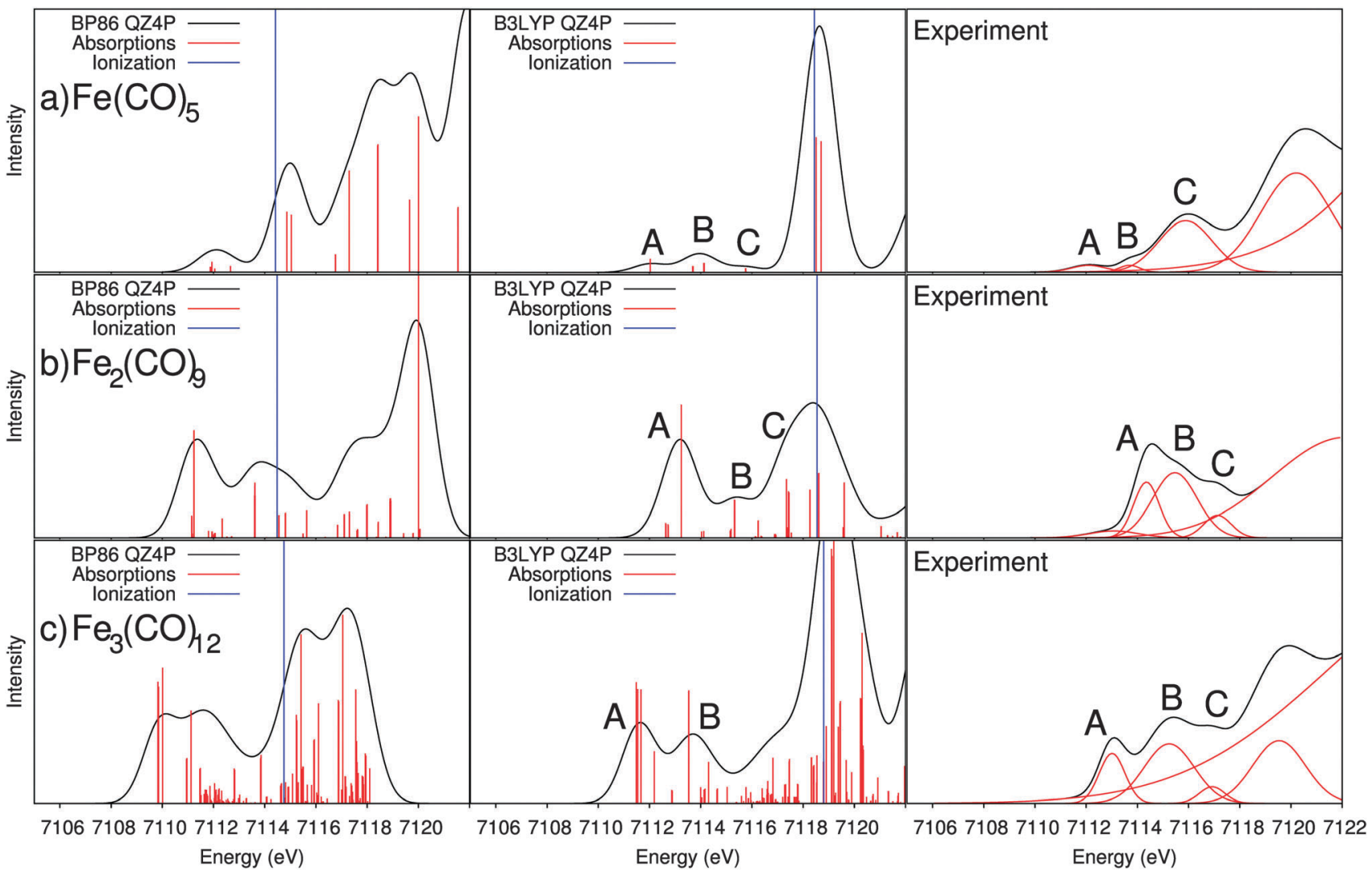

Fig. 4 X-ray absorption spectra of $\mathrm{Fe}(\mathrm{CO})_{5}, \mathrm{Fe}_{2}(\mathrm{CO})_{9}$ and $\mathrm{Fe}_{3}(\mathrm{CO})_{12}$ calculated with the BP86 and B3LYP exchange-correlation functionals and the experimental results.

that are in better agreement with the experimental spectrum. To further understand the differences between BP86 and B3LYP we must consider the transitions that make up the spectra. The single peak in the BP86 spectrum contains twelve contributing transitions lying very close together. Here, the lowest unoccupied orbital is the $E^{\prime \prime}$, but the energy difference to the unoccupied $A_{1}{ }^{\prime}$ orbital is only $0.1 \mathrm{eV}$. All twelve unoccupied orbitals contributing to the pre-edge transitions lie within $2.3 \mathrm{eV}$. Therefore, once the broadening of the transitions is taken into account, only one pre-edge peak remains. Clearly, such a picture of the electronic structure is contradicted by the experimental spectrum.

On the other hand, in the B3LYP spectrum two well-separated peaks appear. The lowest energy transition is to the $A_{1}{ }^{\prime}$ orbitals (which can be assigned to peak A in the experimental spectrum), followed by transitions to the two degenerate representations $E^{\prime \prime}$ and $E^{\prime}$ (which can be assigned to peak B in the experimental spectrum). Here, the energy separation between $A_{1}{ }^{\prime}$ and $E^{\prime \prime}$ is $1.7 \mathrm{eV}$, and the difference between the first and last transition in the pre-edge is $5.4 \mathrm{eV}$. The isosurface plots of the (combinations of) unoccupied orbitals observed in these peaks are shown in Fig. 5a. These agree with the unoccupied orbitals obtained with BP86 (shown in the MO diagram in Fig. 3). However, as the spectrum obtained with B3LYP points to an electronic structure that is in better agreement with experiment, the orbital ordering extracted from the B3LYP spectrum has been used in the MO diagram of Fig. 3.
Table 1 shows the dipole and quadrupole contributions to the intensities of the transitions, separated into the transitions that contribute to each of the peaks. We can see that the contributions for $\mathrm{Fe}(\mathrm{CO})_{5}$ follow what is expected from symmetry considerations: Peak A $\left(A_{1}{ }^{\prime}\right)$ is composed entirely of quadrupole intensity and peak $\mathrm{B}\left(E^{\prime \prime}\right.$ and $\left.E^{\prime}\right)$ has a small dipole contribution due to the transition into the $E^{\prime}$ orbitals. We note that earlier calculations $^{66}$ of the Fe K-edge XAS spectra of $\mathrm{Fe}(\mathrm{CO})_{5}$ did not include quadrupole contributions to the intensities, and thus only showed a single pre-edge peak due to transitions to the $E^{\prime}$ orbitals.

\section{$4 \mathrm{Bi}-$ and trinuclear iron carbonyls}

We now turn to the bi- and trinuclear iron carbonyl complexes $\mathrm{Fe}_{2}(\mathrm{CO})_{9}$ and $\mathrm{Fe}_{3}(\mathrm{CO})_{12}$, which have a molecular structures with $D_{3 \mathrm{~h}}$ and $C_{2 \mathrm{v}}$ symmetry, respectively. ${ }^{19,67,68}$ In $\mathrm{Fe}_{2}(\mathrm{CO})_{9}$, both iron centres have a pseudo-octahedral environment (consisting of five CO ligands and the other iron atom), and three bridging CO ligands connect the two iron centres, while each iron centre features three terminal CO ligands. The molecular structure of $\mathrm{Fe}_{3}(\mathrm{CO})_{12}$ can be derived from $\mathrm{Fe}_{2}(\mathrm{CO})_{9}$ by replacing one bridging $\mathrm{CO}$ ligand by an $\mathrm{Fe}(\mathrm{CO})_{4}$ moiety. Thus, all three iron centres are still in a pseudo-octahedral environment. However, the bonds between the iron atom of the $\mathrm{Fe}(\mathrm{CO})_{4}$ moiety and those of the $\mathrm{Fe}_{2}(\mathrm{CO})_{8}$ backbone are not bridged by $\mathrm{CO}$ ligands anymore. 

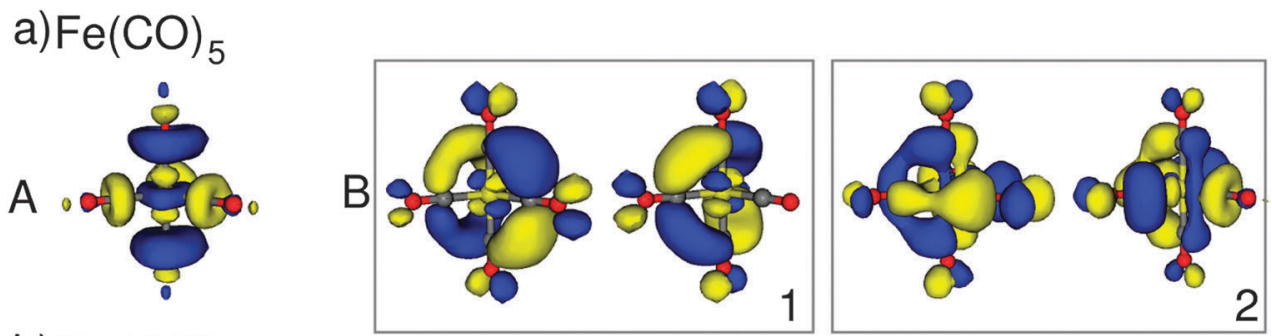

b) $\mathrm{Fe}_{2}(\mathrm{CO})_{9}$

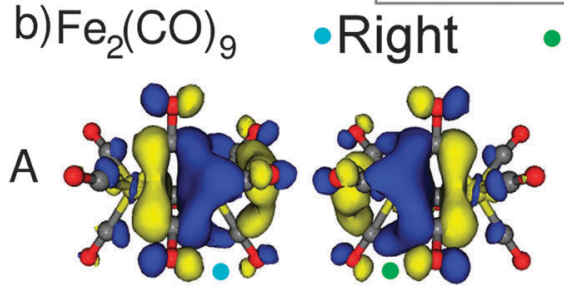

Left
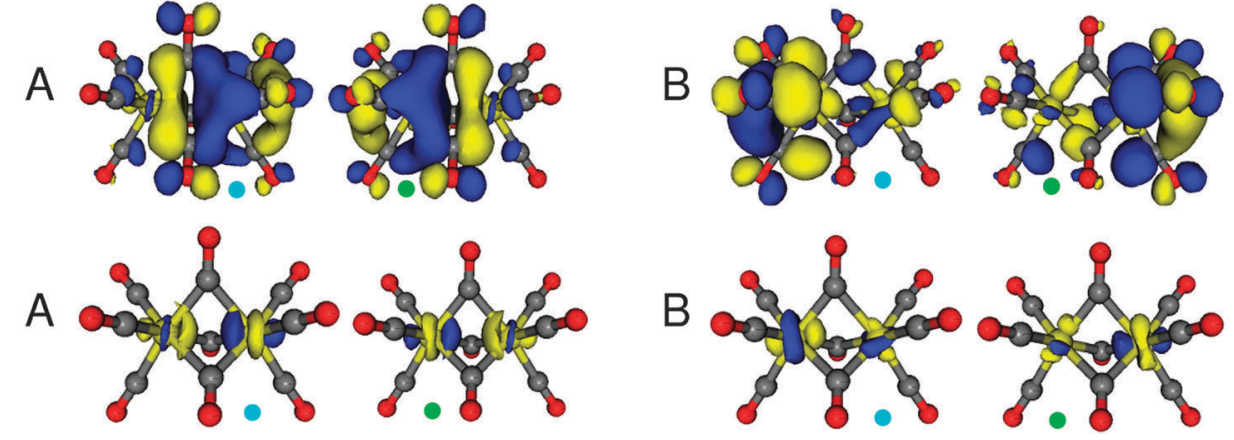

Fig. 5 Isosurface plots of the unoccupied orbitals probed by the pre-edge transitions as obtained from TD-DFT calculations with B3LYP/QZ4P, for the major contributions to the pre-edge peaks of $\mathrm{Fe}(\mathrm{CO})_{5}$ and $\mathrm{Fe}_{2}(\mathrm{CO})_{9}$. Shown below the unoccupied orbitals are the isolated iron orbital contributions to them. The coloured dots indicate the iron atom (1s orbital) from which the transition originates.

Table 1 The percentage of dipole and quadrupole contributions to the transition intensity for the most intense transitions in the calculated B3LYP spectra

\begin{tabular}{llcc}
\hline Complex & Peak & Dipole (\%) & Quadrupole (\%) \\
\hline (a) $\mathrm{Fe}(\mathrm{CO})_{5}$ & $\mathrm{~A}$ & 0.0 & 100.0 \\
& $\mathrm{~B}-1$ & 0.0 & 100.0 \\
& $\mathrm{~B}-2$ & 25.2 & 74.8 \\
& & & \\
(b) $\mathrm{Fe}_{2}(\mathrm{CO})_{9}$ & $\mathrm{~A}$ & 96.3 & 3.7 \\
& $\mathrm{~B}$ & 100.0 & 0.0 \\
& & & \\
(c) $\mathrm{Fe}_{3}(\mathrm{CO})_{12}$ & $\mathrm{~A}-1$ & 92.7 & 7.3 \\
& $\mathrm{~A}-2$ & 78.0 & 22.0 \\
& $\mathrm{~B}-1$ & 95.4 & 4.6 \\
& $\mathrm{~B}-2$ & 88.8 & 11.2 \\
(d) $\mathrm{Fe}(\mathrm{CO})_{3}\left(\mathrm{C}_{8} \mathrm{H}_{12}\right)$ & $\mathrm{A}$ & 87.1 & 12.9 \\
& & & \\
(e) $\mathrm{Fe}_{2}(\mathrm{CO})_{4}(\mathrm{Cp})_{2}$ & $\mathrm{~A}-1$ & 82.6 & 17.4 \\
& $\mathrm{~A}-2$ & 96.0 & 4.0 \\
& $\mathrm{~B}-1$ & 97.6 & 2.4 \\
& $\mathrm{~B}-2$ & 91.5 & 8.5
\end{tabular}

Numerous computational studies of both the geometrical and the electronic structures of these bi- and trinuclear carbonyls have been performed, ${ }^{69-75}$ and the presence of direct metalmetal bonds has been discussed extensively. While applying the 18-electron rules clearly points towards Fe-Fe bonds, their presence is questioned by most theoretical studies for $\mathrm{Fe}-\mathrm{Fe}$ bonds bridged by carbonyl ligands. For $\mathrm{Fe}_{2}(\mathrm{CO})_{9}$, most computational studies rule out a direct $\mathrm{Fe}-\mathrm{Fe}$ bond and attribute the stabilising interactions to the bridging carbonyl ligands $s^{69,70,73}$ or only point to a very weak direct metal-metal interaction. ${ }^{74}$ On the other hand, two of the three Fe-Fe bonds in $\mathrm{Fe}_{3}(\mathrm{CO})_{12}$ are not bridged by carbonyl ligands and must thus be due to a direct metal-metal bonding interaction. ${ }^{71,72}$ Below, we will discuss to what extent our HERFD-XANES spectra can provide indications for these different kinds of metal-metal interactions.

For $\mathrm{Fe}_{2}(\mathrm{CO})_{9}$, the experimental and calculated spectra are shown in Fig. 4b. There are three observable features in the experimental spectrum labelled A, B, and C. The conventional K-edge spectrum of $\mathrm{Fe}_{2}(\mathrm{CO})_{9}$ shows only one distinct peak, again demonstrating the capability of HERFD-XANES. Within the experimental spectra we can assign both peaks A and $\mathrm{B}$ to the pre-edge whereas peak $\mathrm{C}$ is once again most likely due to transitions to Rydberg states or other states close to the absorption edge. Therefore, we will focus on peaks A and B in the following. If we compare the spectrum to the one of $\mathrm{Fe}(\mathrm{CO})_{5}$, we immediately notice that the pre-edge features have a much higher intensity and that all three peaks are shifted to higher energies.

First, we compare the spectra calculated with both the BP86 and B3LYP exchange-correlation functionals. BP86 calculates a spectrum with only two distinct features below the ionisation threshold, whereas B3LYP shows three distinct features. The latter appears to agree better with the experimental observations. Therefore, we will only discuss the B3LYP results in the following. The isosurface plots of the (combination of) unoccupied orbitals that are probed with a significant intensity contribution are plotted in the upper part of Fig. 5b. To highlight the contributions of the iron orbitals, the lower part of Fig. $5 \mathrm{~b}$ shows isosurface plots that only cover a radius of $1 \AA$ around the iron atoms.

These orbital plots show that peak A is comprised of transitions to an anti-bonding combination of the iron $\mathrm{d}_{z^{2}}$-orbitals, which further combines with carbonyl $\pi^{*}$-orbitals. The two contributing excitations originate from each of the two core orbitals to 
a combination of unoccupied orbitals. Both $\mathrm{Fe}(\mathrm{CO})_{5}$ and $\mathrm{Fe}_{2}(\mathrm{CO})_{9}$ have the same point group and the major transitions in peak $\mathrm{A}$ are to orbitals containing the iron $\mathrm{d}_{z^{2}}$-orbitals. The main reason for the large increase of peak $\mathrm{A}$ in intensity is that even though the molecular point group is the same, the symmetry of the iron centres has been reduced. In $\mathrm{Fe}_{2}(\mathrm{CO})_{9}$ neither lie in the horizontal mirror plane and the lowest energy $\mathrm{d}_{z^{2}}$-combination now belongs to the $A_{2}{ }^{\prime \prime}$ irrep, whereas it belongs to the $A_{1}{ }^{\prime}$ irrep in $\mathrm{Fe}(\mathrm{CO})_{5}$. Therefore, the corresponding transition becomes dipole-allowed, which can be seen in Table 1. The shift of peak A to higher energy can be rationalised by a combination of three factors: In $\mathrm{Fe}_{2}(\mathrm{CO})_{9}$, the $\mathrm{d}_{z^{2}}$-orbitals now interact with the $\pi^{*}$ ligand orbitals, which pushes it to higher energies. At the same time, the change in the ligand field of the iron centre from a pentagonal bipyramidal to a pseudo-octahedral ligand field (where the $\mathrm{d}_{z^{2}}$-orbital is not involved in the bonding of the ligands) lowers the energy. Finally, the interaction of the $\mathrm{d}_{z^{2}}$-orbitals of the two iron centres to form an anti-bonding combination increased the energy of the transition. The latter effect can be seen as an indication of direct metal-metal bonding, but is difficult to isolate from the other two (indirect) contributions.

Peak B has a more complicated assignment. From the isosurface plots in Fig. 5 we can see that one of the iron atom orbitals is $\mathrm{d}_{z^{2}}$-like, while the other one belongs to the degenerate pair of iron $\mathrm{d}_{x z}$ and $\mathrm{d}_{y z}$-orbitals. The second transition is a mirror image of the first one, with the phase of the $d_{z^{2}}$-like orbitals reversed. The two transitions each originate from the iron atom upon which the $\mathrm{d}_{x z / y z}$-like orbital resides. Therefore, this can be considered a linear combination of transitions which occur from both the $\mathrm{A}$ and $\mathrm{B}$ peaks of $\mathrm{Fe}(\mathrm{CO})_{5}$.

Finally, we consider $\mathrm{Fe}_{3}(\mathrm{CO})_{12}$ where the experimental and calculated spectra are shown in Fig. 4c. The experimental spectrum once more has three observable features, which we have labelled A, B, and C, and we focus on the pre-edge peaks A and B. If we compare the spectrum to the one of $\mathrm{Fe}_{2}(\mathrm{CO})_{9}$, we can see that the features shift to lower energy and that the intensity of peak $\mathrm{A}$ is reduced, while peak $\mathrm{B}$ has a larger intensity. For the calculated spectra we will only discuss the B3LYP spectrum, which shows the better agreement with experiment.

The isosurface plots of the (combination of) unoccupied orbitals that are probed with a significant intensity contribution are plotted in Fig. 6, along with the corresponding iron orbital contribution. Peak A has four main contributing transitions. The first two transitions are from the two $\mathrm{Fe}_{2}(\mathrm{CO})_{8}$ core orbitals to metal-metal anti-bonding $\sigma^{*}$-orbitals between the $\mathrm{Fe}_{2}(\mathrm{CO})_{8}$ backbone and the $\mathrm{Fe}(\mathrm{CO})_{4}$ moiety. The next transition originates from the 1 s orbital of the $\mathrm{Fe}(\mathrm{CO})_{4}$ group to a completely antibonding orbital, in which all three possible metal-metal interactions are out of phase. These three transitions (labelled " 1 " in Fig. 6) all have a similar character, but originate from the three different iron core orbitals. The final transition (labelled "2") also originates from the $1 \mathrm{~s}$ of the $\mathrm{Fe}(\mathrm{CO})_{4}$ group, but goes to an orbital which resembles an antibonding combination of the iron d-orbital of the $\mathrm{Fe}(\mathrm{CO})_{4}$ group and of those of the $\mathrm{Fe}_{2}(\mathrm{CO})_{8}$ backbone, while the d-orbitals of the iron centres of the $\mathrm{Fe}_{2}(\mathrm{CO})_{8}$ backbone combine in a bonding fashion.
Peak B contains three main transitions, in which orbitals from the $\mathrm{Fe}_{2}(\mathrm{CO})_{8}$ backbone are the main contributor. The first two transitions (labelled “ 1 ") are to $\sigma^{*}$ anti-bonding metalmetal bonding orbitals of the backbone, with only a small orbital inclusion from the third iron centre. The third and final major contributing transition (labelled " 2 ") is to an orbital with a possible bonding interaction between the iron centres of the $\mathrm{Fe}_{2}(\mathrm{CO})_{8}$ backbone.

To understand the changes in spectral intensity compared to $\mathrm{Fe}(\mathrm{CO})_{5}$ and $\mathrm{Fe}_{2}(\mathrm{CO})_{9}$, we must note that symmetry of the molecule is reduced from $D_{3 \mathrm{~h}}$ to $C_{2 \mathrm{v}}$. This reduction in symmetry provides more possibilities for dipole-allowed transitions. Hence, the increase in intensity of peak B can be expected. However, the slight decrease in intensity of peak A compared to peak A of $\mathrm{Fe}_{2}(\mathrm{CO})_{9}$, is not so easy to rationalise. If we look at the contributions of the transition intensities to the peak shown in Table 1 we can see that the main intensity contribution for all transitions is dipole. On the other hand, the amount of quadrupole intensity contribution to the peak has also increased relative to $\mathrm{Fe}_{2}(\mathrm{CO})_{9}$.

The peaks also shift to lower energies than in $\mathrm{Fe}_{2}(\mathrm{CO})_{9}$ and the reason is clear when we consider the molecular orbitals. On the introduction of the $\mathrm{Fe}(\mathrm{CO})_{4}$ group to the $\mathrm{Fe}_{2}(\mathrm{CO})_{8}$ backbone, a third set of d-orbitals are introduced, which can interact with the $\sigma / \sigma^{*}$-orbitals of the $\mathrm{Fe}_{2}(\mathrm{CO})_{8}$ backbone. When these d-orbitals interact, they split into three orbitals, shifting the first available unoccupied orbitals to lower energies. This shift to lower energies is then reflected in the pre-edge peaks.

From all the observations discussed it becomes clear how the introduction of a second iron centre affects the electronic structure. A large part of the differences can be explained through a reduction of the symmetry of the individual iron centres and hence, their individual electronic structures. Moreover, we can see a correspondence between peak $\mathrm{A}$ of $\mathrm{Fe}(\mathrm{CO})_{5}$ and $\mathrm{Fe}_{2}(\mathrm{CO})_{9}$ in that they both consist of iron $\mathrm{d}_{z^{2}}$-orbitals. A subsequent correspondence can be made to peak $\mathrm{A}$ of $\mathrm{Fe}_{3}(\mathrm{CO})_{12}$ in that this peak consists only of $\sigma^{*}$-orbitals, the same as for peak A of $\mathrm{Fe}_{2}(\mathrm{CO})_{9}$. All these features can be related to the unoccupied, anti-bonding (and partly bonding) combinations of the iron d-orbitals, even though it is difficult to isolate the contributions of a direct metalmetal interaction from indirect ones.

\section{Substituted iron carbonyl complexes}

In addition to the pure iron carbonyl complexes, we also consider the effect of substitution of one or more carbonyl groups on the pre-edge X-ray absorption spectra and thus, also the electronic structure. It was previously shown that the substitution of carbonyl ligands by hydrocarbons leads to clear changes in the V2C-XES spectra. Here, we want to investigate whether HERFDXANES can provide complementary information.

First, $\mathrm{Fe}(\mathrm{CO})_{3}\left(\mathrm{C}_{8} \mathrm{H}_{12}\right)$ is a mononuclear iron complex, in which two of the $\mathrm{CO}$ groups in $\mathrm{Fe}(\mathrm{CO})_{5}$ have been replaced by an $\eta^{4}$-coordinated cyclooctadiene ligand. In Fig. 7 we show the experimental and B3LYP-calculated spectra of $\mathrm{Fe}(\mathrm{CO})_{3}\left(\mathrm{C}_{8} \mathrm{H}_{12}\right)$ in comparison to $\mathrm{Fe}(\mathrm{CO})_{5}$. In the experimental spectrum of 


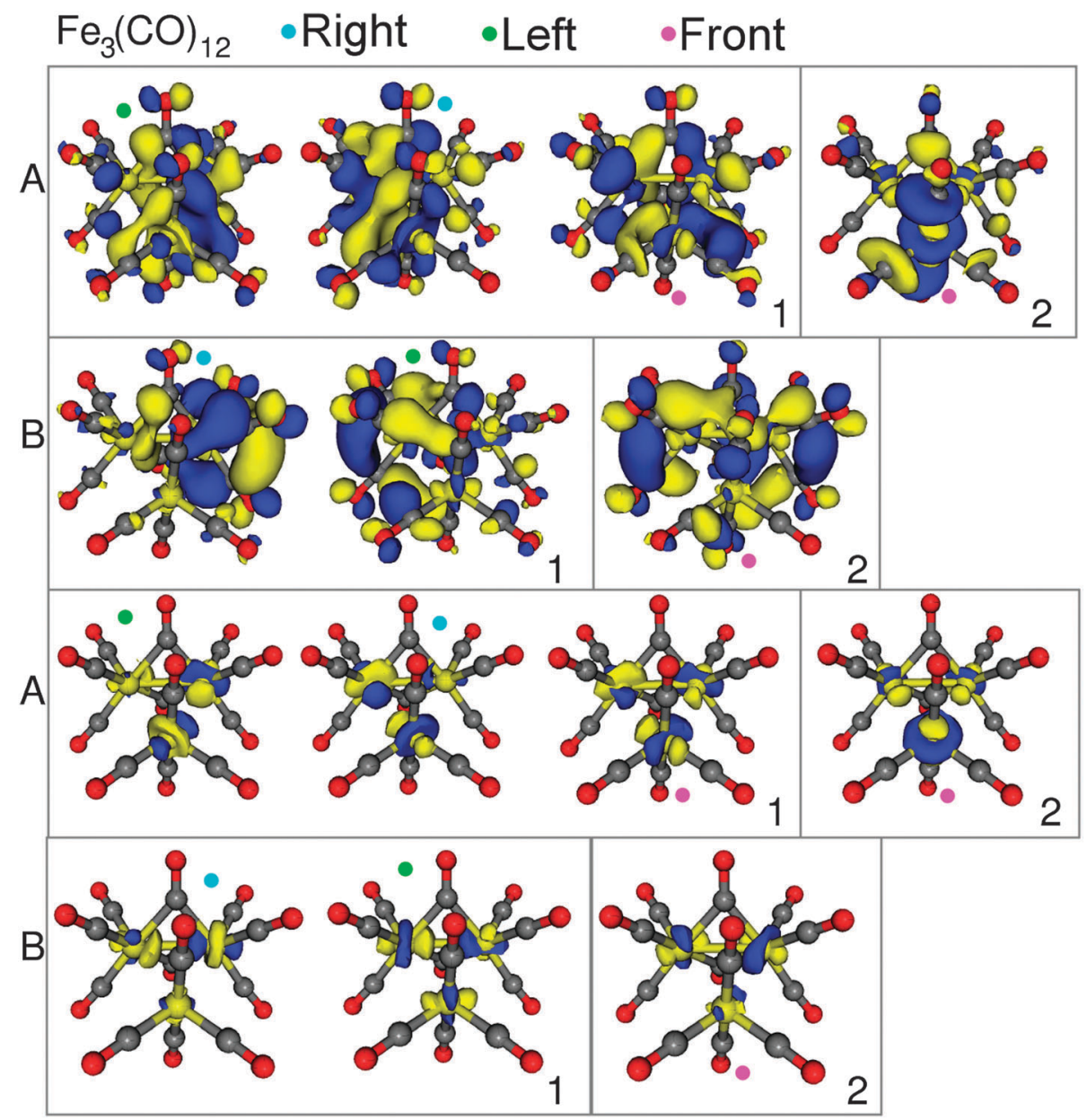

Fig. 6 Isosurface plots of the unoccupied orbitals probed by the pre-edge transitions as obtained from TD-DFT calculations with B3LYP/QZ4P, for the major contributions to the pre-edge peaks of $\mathrm{Fe}_{3}(\mathrm{CO})_{12}$. Shown below the unoccupied orbitals are the isolated iron orbital contributions to them. The coloured dots indicate the iron atom (1s orbital) from which the transition originates.

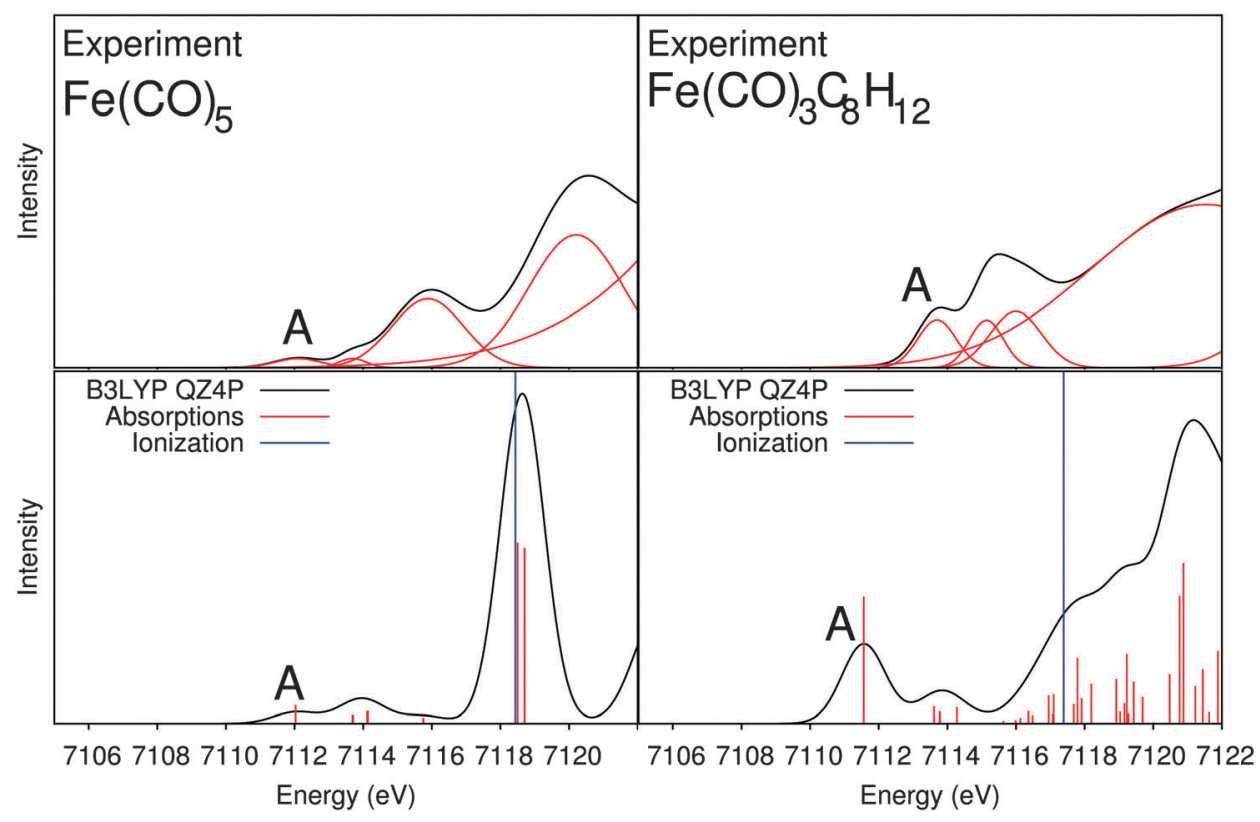

Fig. 7 X-ray absorption spectra for $\mathrm{Fe}(\mathrm{CO})_{5}$ and $\mathrm{Fe}(\mathrm{CO})_{3}\left(\mathrm{C}_{8} \mathrm{H}_{12}\right)$. Top: experimental spectra; bottom: B3LYP calculated spectra. 
a) $\mathrm{Fe}(\mathrm{CO})_{3} \mathrm{C}_{8} \mathrm{H}_{12}$
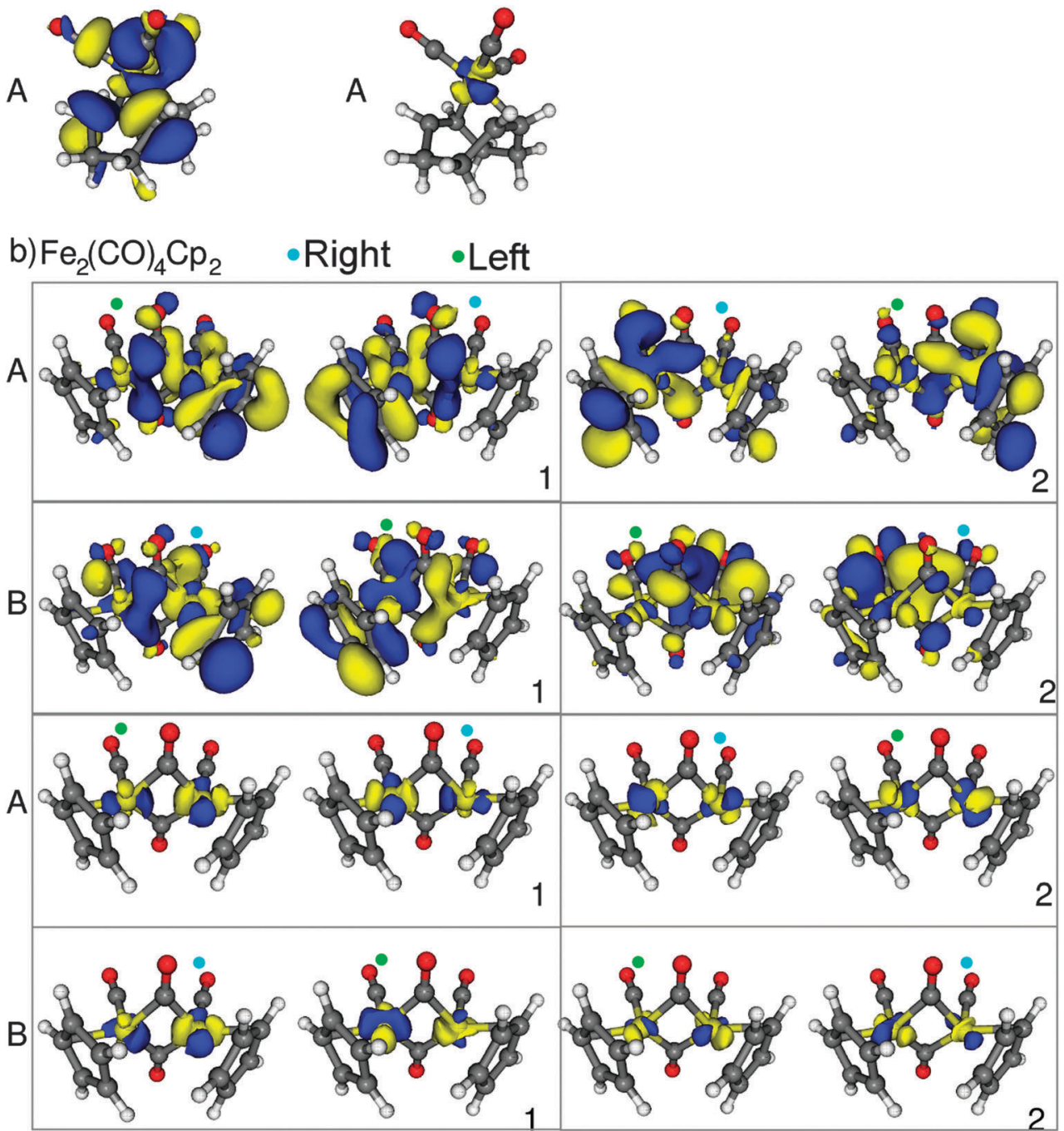

Fig. 8 Isosurface plots of the unoccupied orbitals probed by the pre-edge transitions as obtained from TD-DFT calculations with B3LYP/QZ4P, for the major contributions to the pre-edge peaks of $\mathrm{Fe}(\mathrm{CO})_{3}\left(\mathrm{C}_{8} \mathrm{H}_{12}\right)$ and $\mathrm{Fe}_{2}(\mathrm{CO})_{4}(\mathrm{Cp})_{2}$. Shown below the unoccupied orbitals are the isolated iron orbital contributions to them. The coloured dots indicate the iron atom (1s orbital) from which the transition originates.

$\mathrm{Fe}(\mathrm{CO})_{3}\left(\mathrm{C}_{8} \mathrm{H}_{12}\right)$, we observe one pre-edge feature which has a large intensity increase as well as a shift to higher energies compared to $\mathrm{Fe}(\mathrm{CO})_{5}$. The calculated BP86 spectrum (see ESI $\dagger$ ) shows one peak with a large intensity, while B3LYP leads to two distinct features. In comparison to the experiment, both provide the correct picture with one high-intensity peak. We note that neither exchange-correlation functional reproduces the shift to higher pre-edge peak energies.

To understand what changes occur on substitution of the two carbonyl groups for the $\mathrm{C}_{8} \mathrm{H}_{12}$, we consider the isosurface plots of the unoccupied orbitals that are probed with a significant intensity contribution. These are plotted in Fig. 8 along with the corresponding isolated iron contribution. Peak A of $\mathrm{Fe}(\mathrm{CO})_{3}\left(\mathrm{C}_{8} \mathrm{H}_{12}\right)$ contains a $\mathrm{d}_{x z / y z}$-like orbital, which is an antibonding combination of this iron orbital and $\pi^{*}$-orbitals of the $\mathrm{C}_{8} \mathrm{H}_{12}$ ligand. For $\mathrm{Fe}(\mathrm{CO})_{5}$, the transition to the corresponding $\mathrm{d}_{x z / y z}$-orbitals were contained in the second pre-edge peak (peak B).
This can be used to rationalise the shift to a higher energy, even though this shift is not reproduced correctly by the calculations. The loss of the degeneracy of the $\mathrm{d}_{x z / y z}$-orbitals can be explained through its interaction with the $\mathrm{C}_{8} \mathrm{H}_{12}$ ligand, because only one of the two $\mathrm{d}_{x z / y z}$-orbitals will be in the correct orientation to interact with the $\pi^{*}$-orbitals of the $\mathrm{C}_{8} \mathrm{H}_{12}$ ligand. The large increase in intensity can be attributed to the reduction of the symmetry at the iron centre. We can see that in Table 1 that the primary intensity contribution is now due to electricdipole contributions.

We also considered a bimetallic substituted iron carbonyl system, $\mathrm{Fe}_{2}(\mathrm{CO})_{4}(\mathrm{Cp})_{2}$, where five of the $\mathrm{CO}$ groups in $\mathrm{Fe}_{2}(\mathrm{CO})_{9}$ have been replaced by two cyclopentadiene groups (one on each iron centre). Fig. 9 shows the experimental and B3LYP-calculated spectra of $\mathrm{Fe}_{2}(\mathrm{CO})_{4}(\mathrm{Cp})_{2}$ and of $\mathrm{Fe}_{2}(\mathrm{CO})_{9}$ for comparison. In the experimental spectrum, we observe two distinguishable pre-edge features, labelled A and B. Compared to the spectrum of $\mathrm{Fe}_{2}(\mathrm{CO})_{9}$ 


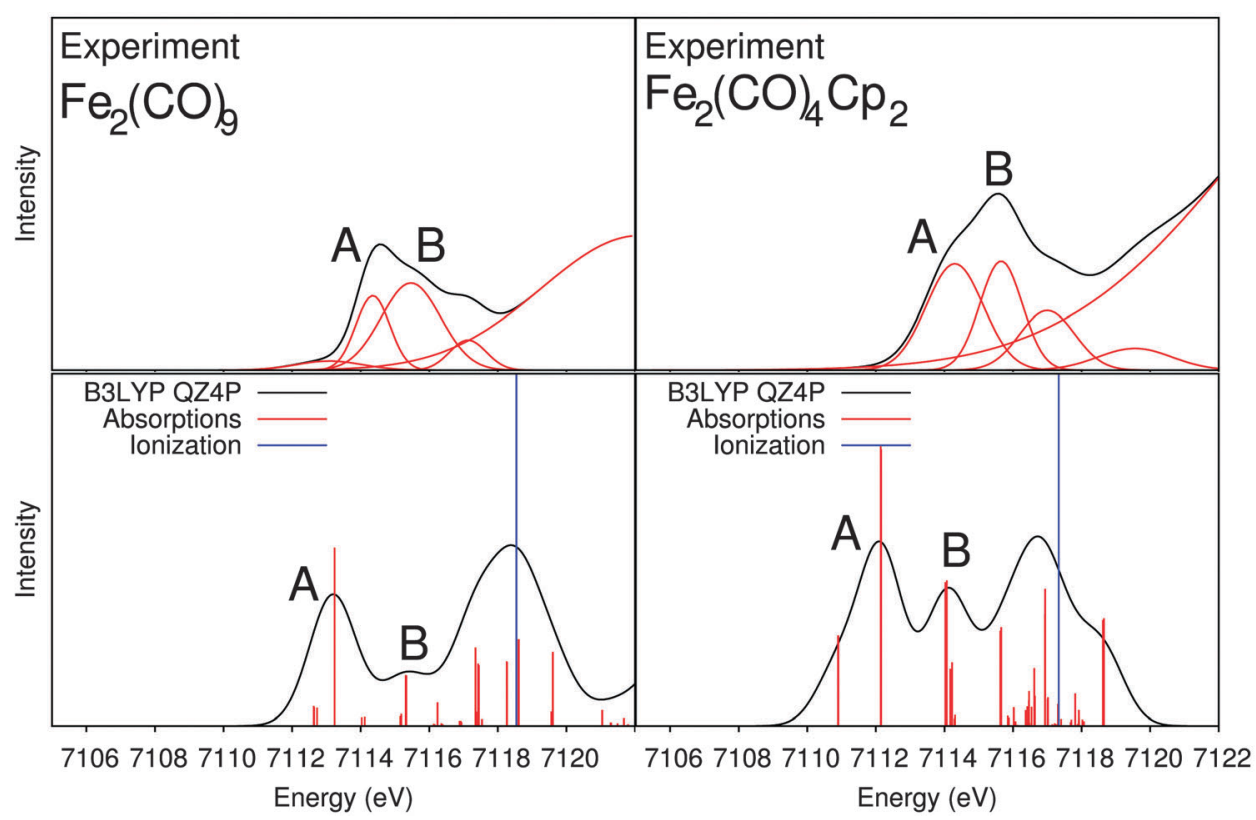

Fig. 9 X-ray absorption spectra for $\mathrm{Fe}_{2}(\mathrm{CO})_{9}$ and $\mathrm{Fe}_{2}(\mathrm{CO})_{4}(\mathrm{Cp})_{2}$. Top: experimental spectra; bottom: B3LYP calculated spectra.

no significant shift in the peak positions is observed, but there is a significant increase in the intensity of both features. Again, the spectra calculated with B3LYP are more accurate compared to the experimental spectrum than the ones obtained with BP86 (see ESI $\dagger$ ).

The isosurface plots of the unoccupied orbitals that are probed with a significant intensity contribution are plotted in Fig. 8 along with the corresponding isolated iron orbital contribution. Peak A is comprised of four transitions, two from each core orbital. The first two transitions (labelled "1") are to orbitals which are antibonding combinations of $\mathrm{d}_{x z / y z}$-like orbitals on the two iron centres interacting in an anti-bonding fashion with $\pi^{*}$-orbitals of the Cp-ligand at one of the iron atoms. The other two transitions (labelled " 2 ") are once again to $\mathrm{d}_{x z / y z}$-like iron orbitals interacting in an anti-bonding fashion with other $\mathrm{Cp} \pi^{*}$-orbitals, which are rotated by approximately 90 degrees around the $\mathrm{Cp}$ ring. Peak B of $\mathrm{Fe}_{2}(\mathrm{CO})_{4} \mathrm{Cp}_{2}$ also contains four main transitions, two from each core orbital. The first two transitions (labelled " 1 ") are to orbitals with anti-bonding characteristics between a $\mathrm{d}_{x z / y z}$-like orbital and the $\mathrm{Cp} \pi^{*}$-orbitals on one of the irons. However, unlike in peak A, the other iron atom now has a $\mathrm{d}_{z^{2}}$-like appearance. The final two transitions (labelled " 2 ") are $\mathrm{d}_{x^{2}-y^{2} / x y}$-like for the iron orbitals and from Fig. 8 appear to be anti-bonding orbitals for the metal-CO bonds.

The large increase in the intensity of both pre-edge peaks in relation to the pre-edge peaks of $\mathrm{Fe}_{2}(\mathrm{CO})_{9}$ can be explained through the decrease in the symmetry of the complex. The same as was observed for $\mathrm{Fe}(\mathrm{CO})_{3}\left(\mathrm{C}_{8} \mathrm{H}_{12}\right)$. We can see in Table 1 that the intensity is mainly due to dipole contributions. However, as the $\mathrm{Fe}_{2}(\mathrm{CO})_{9}$ peaks were also primarily composed of dipole transitions, only the loss of symmetry and thus, relaxation of the selection rules for the dipole mechanism can account for the increase in the intensity.
In conclusion, we can see that substitution of carbonyl groups for a group containing $\pi$-orbitals reduces the symmetry and thus, the intensity of the spectra increases. In addition, the inclusion of $\pi$-orbital containing substituents increases the intensity of transitions to the $\mathrm{d}_{x z / y z}$-like iron orbitals, which can combine with the ligand $\pi^{*}$-orbitals, compared to the $\mathrm{d}_{z^{2}}$-like iron orbital(s) that dominated for the pure iron carbonyl complex spectra.

\section{Conclusions}

The HERFD-XANES spectra of iron carbonyl model complexes reported here demonstrate the unique sensitivity of the experimental method to changes both in the geometric and in the electronic structure at the iron centre(s). By comparison with quantum-chemical calculations, the pre-edge region of the spectra can be analysed in detail, which provides a clear assignment of the observed spectral features and allows us to relate changes in the pre-edge peaks to changes in the electronic structure of the complexes.

We note here that the two exchange-correlation functionals considered, BP86 and B3LYP, lead to XANES spectra that differ significantly. Similar observations have been made before by us and others. ${ }^{33,49,76}$ For the example at hand, we found that B3LYP was sufficiently accurate to allow for a qualitative assignment and analysis of the experimental spectra. However, significant deviations between the spectra calculated with B3LYP and the experimental spectra remain, both for relative energies and relative intensities when comparing different complexes. Therefore, we have not performed a quantitative comparison of the experimental and calculated spectra. This situation is clearly not satisfactory, and calls for a more detailed assessment of various (TD-)DFT 
approaches and of different exchange-correlation functionals for the calculation of X-ray absorption spectra. Moreover, the development of accurate wave-function based methods for X-ray spectroscopy that are still applicable to multi-nuclear transition metal complexes appears promising. ${ }^{77,78}$

Nevertheless, the combination of the experimental HERFDXANES spectra and our TD-DFT calculations using the B3LYP exchange-correlation functional demonstrates the advantages of HERFD-XANES for spectroscopic studies on multinuclear iron complexes. First, it is possible to distinguish the different types of iron-iron bonding interactions. For the pure iron carbonyl complexes, we have shown that the pre-edge region shows different signatures for the mono-, bi-, and trimetallic iron carbonyls. Interestingly, the V2C-XES spectra of these compounds show only subtle differences. ${ }^{25}$ Our assignment shows that the first pre-edge peak $A$ is in all cases due to $\mathrm{d}_{z^{2}}$-like orbitals, while the second peak B is due to $\mathrm{d}_{x y / y z}$-like orbitals. For the bi- and trinuclear complexes, the d-orbitals of the different iron centres form bonding and antibonding combinations (which are also mediated by the interaction with the ligand $\pi^{*}$-orbitals), which modify their orbital energies and the corresponding intensities. Therefore, these peaks both reflect the change in the coordination environment of the iron centres and the change in the iron-iron interactions. An isolated iron centre in $\mathrm{Fe}(\mathrm{CO})_{5}$, a CO-bridged iron-iron bond in $\mathrm{Fe}_{2}(\mathrm{CO})_{9}$ and the direct iron-iron bonds in $\mathrm{Fe}_{3}(\mathrm{CO})_{12}$ can be clearly distinguished, even though the iron has the same oxidation state in all three complexes.

Such a distinction remains possible if some of the carbonyl ligands are replaced by hydrocarbons (cyclopentadienyl and cyclooctadienyl). In this case, the intensities of the pre-edge peaks increase, but they are largely unmodified with respect to the underlying pure carbonyl complexes. In contrast, in the V2C-XES spectra ${ }^{25}$ such an exchange of carbonyl ligands leads to the appearance of new peaks, i.e., it can clearly detect the identity of the ligands. Thus, HERFD-XANES and V2C-XES provide complementary information that, in combination, will make it possible to detect the coordination environment, different types of iron-iron interactions, and the identity of the ligands. Therefore, these methods offer a unique potential for in situ studies following both the ligand environment and the electronic structure of iron carbonyls during catalytic processes.

\section{Acknowledgements}

We thank the ESRF and staff of beamline ID26 for provision and support during the measurements. The Deutsche Forschungsgemeinschaft is acknowledged for funding through FOR 1405 (MB) and the DFG-Center for Functional Nanostructures at KIT (AJA and CRJ).

\section{References}

1 F. A. Cotton, G. Wilkinson, C. A. Murillo and M. Bochmann, Advanced Inorganic Chemistry, Wiley-Interscience, New York, 6th edn, 1999.
2 N. Wiberg, E. Wiberg and A. F. Holleman, Lehrbuch der Anorganischen Chemie, Gruyter, Berlin, New York, Auflage: 102., stark umgearb. u. verb. edn, 2007.

3 C. Elschenbroich, Organometallics, Wiley-VCH, Weinheim, 3rd edn, 2006.

4 A. Chen, M. Kaminsky, G. L. Geoffroy and M. A. Vannice, J. Phys. Chem., 1986, 90, 4810-4819.

5 M. Kollár, A. De Stefanis, H. E. Solt, M. R. Mihályi, J. Valyon and A. A. G. Tomlinson, J. Mol. Catal. A: Chem., 2010, 333, 37-45.

6 C. Li, P. Wang, W. Ying and D. Fang, Pet. Sci. Technol., 2011, 29, 1546-1554.

7 H. P. Withers, K. F. Eliezer and J. W. Mitchell, Ind. Eng. Chem. Res., 1990, 29, 1807-1814.

8 S. Lögdberg, M. Lualdi, S. Järs, J. C. Walmsley, E. A. Blekkan, E. Rytter and A. Holmen, J. Catal., 2010, 274, 84-98.

9 C. Zhang, G. Zhao, K. Liu, Y. Yang, H. Xiang and Y. Li, J. Mol. Catal. A: Chem., 2010, 328, 35-43.

10 B. H. Davis, Catal. Today, 2009, 141, 25-33.

11 R. Schoch, H. Huang, V. Schünemann and M. Bauer, ChemPhysChem, 2014, 15, 3768-3775.

12 M. Tepluchin, M. Casapu, A. Boubnov, H. Lichtenberg, D. Wang, S. Kureti and J.-D. Grunwaldt, ChemCatChem, 2014, 6, 1763-1773.

13 H. Kuroda, Pure Appl. Chem., 1992, 64, 14491460.

14 P. Johnston, G. J. Hutchings, N. J. Coville, K. P. Finch and J. R. Moss, Appl. Catal., A, 1999, 186, 245-253.

15 F. Gärtner, B. Sundararaju, A.-E. Surkus, A. Boddien, B. Loges, H. Junge, P. H. Dixneuf and M. Beller, Angew. Chem., Int. Ed., 2009, 48, 9962-9965.

16 R. Jennerjahn, R. Jackstell, I. Piras, R. Franke, H. Jiao, M. Bauer and M. Beller, ChemSusChem, 2012, 5, 734-739.

17 M. Wrighton, Chem. Rev., 1974, 74, 401-430.

18 P. T. Snee, C. K. Payne, S. D. Mebane, K. T. Kotz and C. B. Harris, J. Am. Chem. Soc., 2001, 123, 6909-6915.

19 E. Hunstock, C. Mealli, M. J. Calhorda and J. Reinhold, Inorg. Chem., 1999, 38, 5053-5060.

20 W. F. Edgell, W. E. Wilson and R. Summitt, Spectrochim. Acta, 1963, 19, 863-872.

21 L. Bañares, T. Baumert, M. Bergt, B. Kiefer and G. Gerber, Chem. Phys. Lett., 1997, 267, 141-148.

22 C. Crotti, E. Farnetti, T. Celestino and S. Fontana, J. Electron Spectrosc. Relat. Phenom., 2003, 128, 141-157.

23 C. Crotti, E. Farnetti, T. Celestino, M. Stener and S. Fontana, Organometallics, 2004, 23, 5219-5225.

24 M. Bauer and H. Bertagnolli, in Methods in Physical Chemistry, ed. R. Schäfer and P. C. Schmidt, Wiley-VCH Verlag GmbH \& Co. KGaA, 2012, pp. 231-269.

25 M. U. Delgado-Jaime, S. DeBeer and M. Bauer, Chem. - Eur. J., 2013, 19, 15888-15897.

26 M. F. Ruiz-Lopez, M. Loos, J. Goulon, M. Benfatto and C. R. Natoli, Chem. Phys., 1988, 121, 419-437.

27 M. Bauer, T. Kauf, J. Christoffers and H. Bertagnolli, Phys. Chem. Chem. Phys., 2005, 7, 2664-2670.

28 M. Bauer and C. Gastl, Phys. Chem. Chem. Phys., 2010, 12, 5575-5584. 
29 P. Eisenberger, P. M. Platzman and H. Winick, Phys. Rev. Lett., 1976, 36, 623-626.

30 W. M. Heijboer, P. Glatzel, K. R. Sawant, R. F. Lobo, U. Bergmann, R. A. Barrea, D. C. Koningsberger, B. M. Weckhuysen and F. M. F. de Groot, J. Phys. Chem. B, 2004, 108, 10002-10011.

31 O. V. Safonova, M. Tromp, J. A. van Bokhoven, F. M. F. de Groot, J. Evans and P. Glatzel, J. Phys. Chem. B, 2006, 110, 16162-16164.

32 P. Glatzel, M. Sikora, G. Smolentsev and M. FernándezGarcía, Catal. Today, 2009, 145, 294-299.

33 A. J. Atkins, Ch. R. Jacob and M. Bauer, Chem. - Eur. J., 2012, 18, 7021-7025.

34 A. J. Atkins, M. Bauer and Ch. R. Jacob, Phys. Chem. Chem. Phys., 2013, 15, 8095-8105.

35 H. H. Johann, Z. Phys., 1931, 69, 185-206.

36 T. Johansson, Z. Phys., 1933, 82, 507-528.

37 C. Gauthier, V. A. Solé, R. Signorato, J. Goulon and E. Moguiline, J. Synchrotron Radiat., 1999, 6, 164-166.

38 Theoretical Chemistry, Vrije Universiteit Amsterdam, ADF, Amsterdam density functional program, http:/www.scm.com.

39 G. te Velde, F. M. Bickelhaupt, E. J. Baerends, C. Fonseca Guerra, S. J. A. van Gisbergen, J. G. Snijders and T. Ziegler, J. Comput. Chem., 2001, 22, 931-967.

40 A. D. Becke, Phys. Rev. A: At., Mol., Opt. Phys., 1988, 38, 3098-3100.

41 J. P. Perdew, Phys. Rev. B: Condens. Matter Mater. Phys., 1986, 33, 8822-8824.

42 E. Van Lenthe and E. J. Baerends, J. Comput. Chem., 2003, 24, 1142-1156.

43 M. Stener, G. Fronzoni and M. de Simone, Chem. Phys. Lett., 2003, 373, 115-123.

44 K. Ray, S. DeBeer George, E. I. Solomon, K. Wieghardt and F. Neese, Chem. - Eur. J., 2007, 13, 2783-2797.

45 S. DeBeer George, T. Petrenko and F. Neese, Inorg. Chim. Acta, 2008, 361, 965-972.

46 P. Banerjee, S. Sproules, T. Weyhermüller, S. DeBeer George and K. Wieghardt, Inorg. Chem., 2009, 48, 5829-5847.

47 P. Chandrasekaran, S. C. E. Stieber, T. J. Collins, J. Lawrence Que, F. Neese and S. DeBeer, Dalton Trans., 2011, 40, 11070-11079.

48 C. C. Scarborough, S. Sproules, T. Weyhermüller, S. DeBeer and K. Wieghardt, Inorg. Chem., 2011, 50, 12446-12462.

49 M. Roemelt, M. A. Beckwith, C. Duboc, M.-N. Collomb, F. Neese and S. DeBeer, Inorg. Chem., 2012, 51, 680-687.

50 C. C. Scarborough, S. Sproules, C. J. Doonan, K. S. Hagen, T. Weyhermüller and K. Wieghardt, Inorg. Chem., 2012, 51, 6969-6982.

51 C. Lee, W. Yang and R. G. Parr, Phys. Rev. B: Condens. Matter Mater. Phys., 1988, 37, 785-789.

52 A. D. Becke, J. Chem. Phys., 1993, 98, 1372-1377.
53 A. D. Becke, J. Chem. Phys., 1993, 98, 5648-5652.

54 S. Bernadotte, A. J. Atkins and Ch. R. Jacob, J. Chem. Phys., 2012, 137, 204106.

55 S. DeBeer George, T. Petrenko and F. Neese, J. Phys. Chem. A, 2008, 112, 12936-12943.

56 E. Epifanovsky, I. Polyakov, B. Grigorenko, A. Nemukhin and A. I. Krylov, J. Chem. Theory Comput., 2009, 5, 1895-1906.

57 U. Varetto, Molekel, Swiss National Supercomputing Centre, Manno (Switzerland), 2009.

58 E. J. Baerends, C. Oudshoorn and A. Oskam, J. Electron Spectrosc. Relat. Phenom., 1975, 6, 259-267.

59 M. Kotzian, N. Roesch, H. Schroeder and M. C. Zerner, J. Am. Chem. Soc., 1989, 111, 7687-7696.

60 D. Guenzburger, E. Baggio-Saitovitch, M. A. D. Paoli and H. Manela, J. Chem. Phys., 1984, 80, 735-744.

61 R. Fukuda, M. Ehara, H. Nakatsuji, N. Kishimoto and K. Ohno, J. Chem. Phys., 2010, 132, 084302.

62 B. Ahr, M. Chollet, B. Adams, E. M. Lunny, C. M. Laperle and C. Rose-Petruck, Phys. Chem. Chem. Phys., 2011, 13, 5590-5599.

63 M. Bauer, T. Stalinski and E. F. Aziz, ChemPhysChem, 2011, 12, 2088-2091.

64 R. van Leeuwen and E. J. Baerends, Phys. Rev. A: At., Mol., Opt. Phys., 1994, 49, 2421-2431.

65 P. R. T. Schipper, O. V. Gritsenko, S. J. A. van Gisbergen and E. J. Baerends, J. Chem. Phys., 2000, 112, 1344-1352.

66 G. Fronzoni, P. Decleva and A. Lisini, Chem. Phys., 1993, 174, 57-70.

67 F. A. Cotton and J. M. Troup, J. Am. Chem. Soc., 1974, 96, 4155-4159.

68 J. H. Jang, J. G. Lee, H. Lee, Y. Xie and H. F. Schaefer, J. Phys. Chem. A, 1998, 102, 5298-5304.

69 W. Heijser, E. J. Baerends and P. Ros, Faraday Symp. Chem. Soc., 1980, 14, 211-234.

70 C. W. Bauschlicher, Jr., J. Chem. Phys., 1986, 84, 872-875.

71 J. Li and K. Jug, Inorg. Chim. Acta, 1992, 196, 89-95.

72 D. Braga, F. Grepioni, E. Tedesco, M. J. Calhorda and P. E. M. Lopes, J. Chem. Soc., Dalton Trans., 1995, 3297-3306.

73 J. Reinhold, A. Barthel and C. Mealli, Coord. Chem. Rev., 2003, 238-239, 333-346.

74 J. Reinhold, O. Kluge and C. Mealli, Inorg. Chem., 2007, 46, 7142-7147.

75 P. Hirva, M. Haukka, M. Jakonen and M. A. Moreno, J. Mol. Model., 2008, 14, 171-181.

76 F. A. Lima, R. Bjornsson, T. Weyhermüller, P. Chandrasekaran, P. Glatzel, F. Neese and S. DeBeer, Phys. Chem. Chem. Phys., 2013, 15, 20911-20920.

77 J. Wenzel, M. Wormit and A. Dreuw, J. Comput. Chem., 2014, 35, 1900-1915.

78 J. Wenzel, M. Wormit and A. Dreuw, J. Chem. Theory Comput., 2014, 10, 4583-4598. 